\title{
Impacto do exercício físico em idosos com baixo peso: uma abordagem focada na sarcopenia
}

\author{
Impact of physical exercise on low body weight in elderly: \\ a focused approach on sarcopenia
}

\author{
Impacto del ejercicio físico en ancianos con bajo peso: \\ un enfoque centrado en la sarcopenia
}

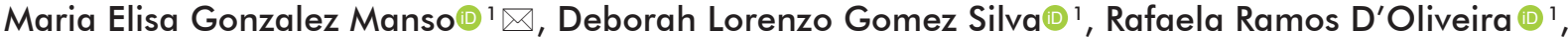
Beatriz Myung Jin Kim (1) ', Ana Flávia de Lima Santos (1) ', Eduardo Antonio Barros Silva Junior (1) '
\end{abstract}

Centro Universitário São Camilo (CUSC)). São Paulo, SP, Brasil.

\begin{tabular}{l} 
ARTICLE INFO \\
\hline Article history \\
Received: 09/02/2019 \\
Accepted: 25/06/2019 \\
Published: 29/08/2019 \\
\hline M Correspondent Author \\
Maria Elisa Gonzalez Manso \\
Av. Nazaré, 1501 - Ipiranga \\
04263-200, São Paulo, SP, Brasil \\
mansomeg@hotmail.com \\
\hline C 2019 All rights reserved \\
\hline Editors \\
Alfredo Cataldo Neto \\
Newton Luiz Terra \\
Assistant Editors \\
Paula Engroff \\
Vanessa Sgnaolin
\end{tabular}

\begin{abstract}
RESUMO
OBJETIVO: Verificar o impacto da prática de exercício físico em idosos com baixo peso e sarcopenia, visto que este último é um componente frequentemente associado à magreza na população idosa. Da mesma forma, busca eliciar os efeitos de um estilo de vida sedentário sobre estas mesmas variáveis.

DELINEAMENTO METODOLÓGICO: Revisão integrativa nas bases PubMed, Scielo e Lilacs no período entre 2014 e 2019 utilizando Descritores em Ciência e Saúde (DeCS) (sarcopenia, idoso, perda de peso, exercício e estilo de vida sedentário) e Medical Subject Headings (MeSH) (aged, aged 80 and over, weight loss, sarcopenia, thinness, exercise e sedentary behaviour).

RESULTADOS: Quatorze artigos foram incluídos na síntese. O estilo de vida sedentário pode levar a perda de força e massa muscular em idosos. Idosos que praticavam atividade física melhoraram o desempenho e a massa muscular, a capacidade funcional e a força.

CONCLUSÃO: Em geral, os artigos demonstraram que a atividade física auxilia na manutenção da massa corporal magra e na potencialização do anabolismo protéico, na população idosa e sarcopênica, além de ser fator preventivo.
\end{abstract}

DESCRITORES: Idoso; Perda de peso; Sarcopenia; Exercício físico.

\begin{abstract}
OBJECTIVE: Verify the impact of physical exercise practice in elders with low weight and sarcopenia, since the latter is a component often associated with thinness in the elderly population. In the same way, it seeks to elicit the effects of a sedentary lifestyle on these same variables.

METHODOLOGICAL DELINEATION: Integrative review of PubMed, Scielo and Lilacs databases between 2014 and 2019 using Descritores em Ciência e Saúde (DeCS) (sarcopenia, idoso, perda de peso, exercício and estilo de vida sedentário) and Medical Subject Headings (MeSH) (aged, aged 80 and over, weight loss, sarcopenia, thinness, exercise and sedentary behavior).

RESULTS: Fourteen articles were included in the synthesis. A sedentary lifestyle can lead to loss of strength and muscle mass among the elderly. Older people who practice physical activity have improved performance and muscle mass, functional capacity, and strength.
\end{abstract}

CONCLUSION: In general, the articles demonstrated that physical activity assists in the maintenance of lean body mass and the potentiation of protein anabolism in the elderly and sarcopenic population, besides being a preventive factor.

KEYwORDS: Aged; Weight loss; Sarcopenia; Physical exercise. 


\section{RESUMEN}

OBJETIVO: Verificar el impacto de la práctica de ejercicio físico por ancianos con bajo peso y sarcopenia, por ser este ultimo un componente frecuentemente asociado a la delgadez en la población anciana. De la misma forma, busca lanzar luz sobre los efectos de un estilo de vida sedentario sobre estas mismas variables.

DELINEAMENTO METODOLÓGICO: Revisión integrativa en las bases PubMed, Scielo y Lilacs, en el periodo comprendido entre los años de 2014 y 2019, utilizando-se Descriptores en Ciencia y Salud (DeCS) (sarcopenia, ancianos, pérdida de peso, ejercicio y estilo de vida sedentario) y Medical Subject Headings (MeSH) (aged, aged 80 and over, weight loss, sarcopenia, thinness, exercise e sedentary behaviour).

RESULTADOS: Catorce artículos se incluyeron en la síntesis. El estilo de vida sedentario puede llevar a la pérdida de fuerza y masa muscular en los ancianos. Los adultos mayores que practicaban actividad física mejoraron el rendimiento y la masa muscular, la capacidad funcional y la fuerza.

CONCLUSIÓN: En general, los artículos demostraran que la actividad física auxilia en el mantenimiento de la masa corporal magra y en la potenciación del anabolismo proteico, en la población anciana y sarcopenica, además de ser un factor de prevención.

Palabras Clave: Anciano; Pérdida de Peso; Sarcopenia; Ejercicio Físico.

\section{INTRODUÇÃO}

O envelhecimento é caracterizado pelo declínio de diversas funções orgânicas, influenciando na capacidade de manutenção da homeostase e acarretando na maior suscetibilidade a doenças. ${ }^{1}$

No que diz respeito às alterações orgânicas, há alterações digestivas, ocasionando na baixa ingestão calórica e protéica e na consequente desnutrição. Há alterações endócrinas, diminuindo a reposição de $\mathrm{GH}$, IGF-1, PTH, testosterona e estrógeno, que interferem na lipólise, síntese de proteínas, na modulação muscular, na quantidade de células satélites e na liberação de citocinas, respectivamente. Além disso, o envelhecimento aumenta os níveis de citocinas pró-inflamatórias, estimulando o processo catabólico de proteínas. ${ }^{2}$

Por fim, há perda de neurônios motores alfa, afetando a coordenação e força muscular, e alterações articulares e ósseas, pela diminuição dos condrócitos e pelo desequilíbrio entre a atividade dos osteoclastos e osteoblastos, respectivamente. Todos estes fatores influenciam direita ou indiretamente na redução da massa e da força muscular, propiciando, portanto, o surgimento de sarcopenia. ${ }^{2}$

A sarcopenia é um processo fisiológico decorrente do envelhecimento, sendo caracterizado por força muscular diminuída, seguida pela baixa qualidade e quantidade muscular, de acordo com o Grupo de Trabalho Europeu sobre Sarcopenia em Idoso 2 (EWGSOP2). ${ }^{3}$

A sua detecção precoce previne o risco elevado de quedas, fraturas, hospitalizações, dependência e mortalidade, por estar relacionada a impactos negativos sobre a saúde dos idosos, como a redução da densidade mineral óssea, a perda de mobilidade, o aumento da fadiga, as disfunções físicas e o aumento do risco cardíaco e metabólico. Para isso, ressalta-se a necessidade de reconhecer os seus fatores de risco, dentre eles, a desnutrição, os medicamentos que causam anorexia, os distúrbios inflamatórios, baixo peso e a inatividade física. ${ }^{4}$

Esta última é de extrema relevância, pois a atividade física aumenta o consumo máximo de oxigênio, estimula a síntese proteica e ativa células satélites, melhorando, consequentemente, a adaptação neuromuscular e a função muscular. ${ }^{2}$ Além de reduzir citocinas pró-inflamatórias e aumentar as antiinflamatórias, reduzindo o catabolismo muscular. ${ }^{5}$

O baixo peso no idoso saudável é determinado pelo IMC $<23 \mathrm{~kg} / \mathrm{m}^{2}$ e está associado ao seu estado nutricional e a sua capacidade funcional, caracterizada pela execução de atividades diárias que envolvem trabalho muscular, de forma segura e independente, sem apresentar fadiga excessiva, incluindo força e resistência muscular, flexibilidade e equilíbrio, podendo ser verificada em testes de desempenho motor. Além disto, o baixo peso em idosos está relacionado a $51 \%$ a mais de óbitos. ${ }^{6}$

Uma vez que o processo de envelhecimento implica em alterações absortivas do TGI e na depleção de fibras musculares, o idoso mau nutrido e com baixo peso está sujeito a potencialização destes processos. ${ }^{1}$ Estes fatores podem levar ao maior risco de morbimortalidade por quedas e fraturas. $^{7}$

A presente revisão objetivou verificar o impacto da prática de exercício físico em idosos com baixo peso e sarcopenia, visto que este último é um componente frequentemente associado à magreza na população idosa. Da mesma forma, busca esclarecer os efeitos de um estilo de vida sedentário sobre estas mesmas variáveis.

\section{DELINEAMENTO METODOLÓGICO}

Realizou-se, durante o segundo semestre de 2018 e primeiro mês de 2019, uma revisão integrativa da 
literatura nas bases de dados PubMed, Scielo e Lilacs compreendendo o período de 2014 a 2019.

Os descritores DeCS utilizados foram: sarcopenia, idosos, baixo peso, atividade física, exercício e sedentarismo; e os descritores MeSH: elderly, oldest, weight reduction, sarcopenia, thinness, physical activities e sedentary behaviour.

A combinação de descritores foi efetuada por meio dos operadores booleanos AND e OR.

Foram identificadas 1041 referências, das quais 67 foram selecionadas para análise de elegibilidade. Os demais 974 estudos foram eliminados através dos resumos, títulos, duplicidade na busca e critérios de exclusão, conforme apresentado de forma gráfica na Figura 1. Dentre os 67 artigos selecionados para análise 14 foram incluídos na síntese com base nos critérios de inclusão.

Foram adotados como critérios de inclusão: a) estudos publicados entre o ano de 2014 e o mês de Janeiro de 2019; b) artigos relacionados à população idosa com baixo peso e à prática de exercício físico; c) artigos relacionados à população idosa com sarcopenia e à prática de exercício físico; d) artigos relacionados aos efeitos da atividade física no organismo do idoso; e) artigos relacionados aos efeitos do estilo de vida sedentário no idoso; f) artigos de revisão, ensaios clínicos, estudos de prevalência, estudos de corte e de caso controle; g) idioma em inglês, espanhol e português; h) artigos completos (pagos e gratuitos).

Excluíram-se: a) estudos anteriores ao ano de 2014; b) artigos que não abordavam os critérios de inclusão $b, c, d$ e $e ; c)$ estudos com modelos experimentais animais; d) séries de casos, relatos de caso e opiniões de especialistas.

\section{RESULTADOS}

Os 14 estudos incluídos foram sumarizados na Tabela 1 quanto aos autores, tamanho e características da amostra, objetivo, método e resultados principais.

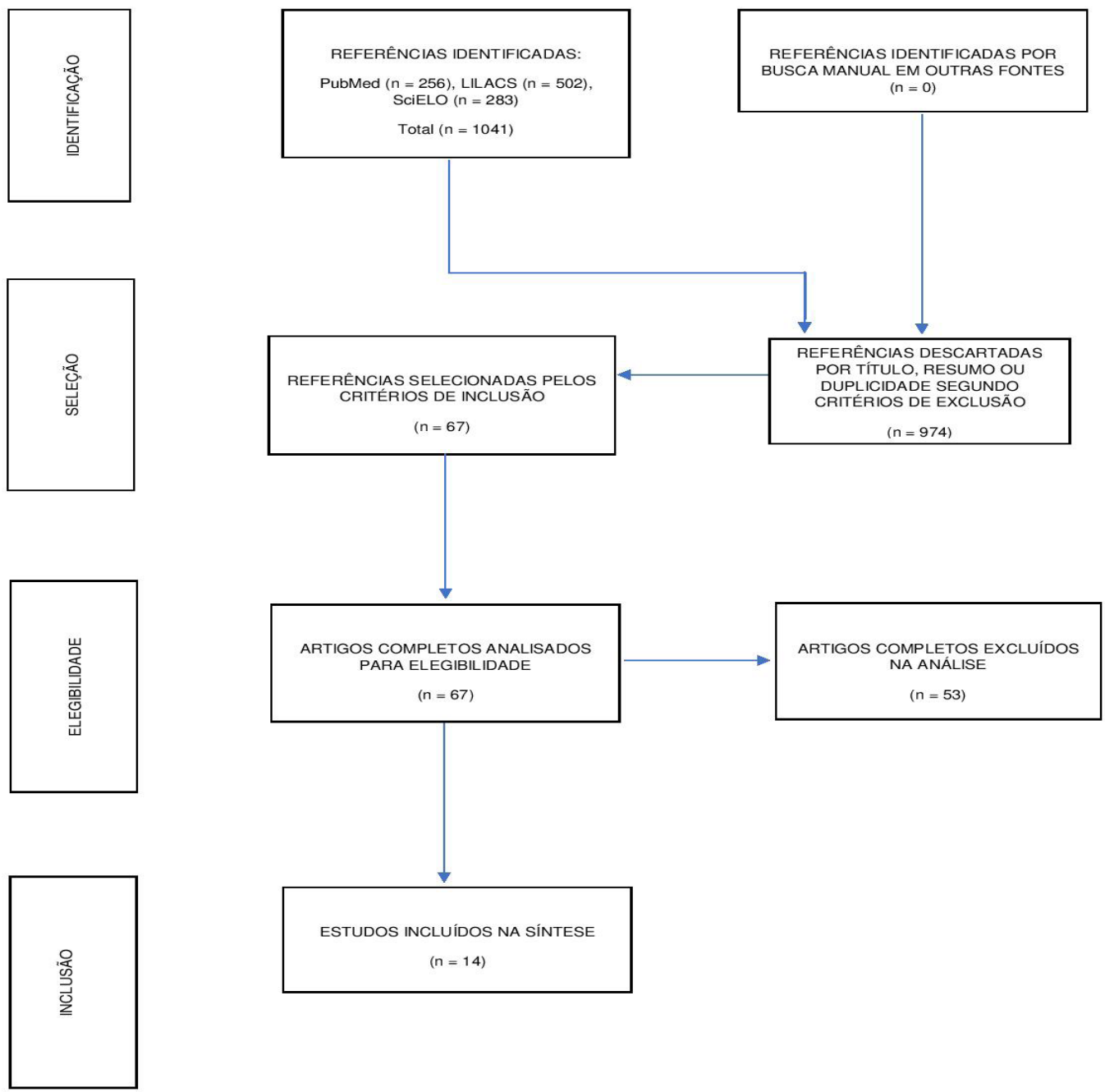

Figura 1. Fluxograma da seleção de artigos nas bases de dados PubMed, LILACS e SciELO no período de 2014 a 2019, com a temática dos descritores: Idoso; Perda de Peso; Sarcopenia; Exercício. 
Tabela 1. Sumário dos Artigos obtidos pela revisão, período de 2014 a 2019, com a temática dos descritores: Idoso; Perda de Peso; Sarcopenia; Exercício.

\begin{tabular}{llll}
\hline Autor e ano & Amostra & Objetivo & Método \\
Magistro D, Candela F, & $\begin{array}{l}202 \text { indivíduos } \\
\text { Brustio PR et al., 2015 }\end{array}$ & $\begin{array}{l}\text { Examinar a relação e } \\
\text { sedentários }(>65 \text { anos). }\end{array}$ & $\begin{array}{l}\text { Estudo longitudinal com três } \\
\text { braços de pesquisa. Todos os } \\
\text { resistência aeróbica e força } \\
\text { participantes foram testados três } \\
\end{array}$ \\
& & $\begin{array}{l}\text { corporal inferior em idosos } \\
\text { sedentários italianos ao longo } \\
\text { do tempo. }\end{array}$ & $\begin{array}{l}\text { partir dos testes de resistência } \\
\text { aeróbica e força corporal } \\
\text { retirados da bateria Senior } \\
\end{array}$ \\
& & Fitness Test.
\end{tabular}

Tanner RE, Brunker LB, 23 indivíduos

Agergaard J et al., 2015 saudáveis. Sendo 14 jovens (18-35 anos) e 9 idosos (60-75 anos).

Calado LB, Ferriolli E, 385 idosos Moriguti JC et. al., 2015 (média $73.9 \pm 6.5$ anos)
Avaliar se há diferenças relacionadas a idade na massa muscular e as respostas anabólicas e catabólicas musculares durante o repouso no leito e determinar se a reabilitação reverteria esses resultados.

Determinar a prevalência e características da síndrome da fragilidade em uma população urbana de idosos.
Foi realizado um desafio de cinco dias de repouso na cama (BED REST). Seguido por 8 semanas de exercício resistido de alta intensidade (REHAB). Determinou-se a massa magra das pernas (via absortometria de raios X de dupla energia; DXA) e força. Biópsias musculares foram coletadas durante uma infusão constante de isótopos estáveis no estado pós absortivo e após a ingestão de aminoácidos essenciais (EAA) em três ocasiões: antes (PRÉ), após o repouso e após a reabilitação. Além disso, as amostras foram avaliadas quanto à síntese proteica, sinalização mTORC1, Expressão de REDD1/2 e marcadores moleculares relacionados à proteólise muscular (MURF1, MAFBX, AMPKo, LC3II/I, Beclin1).

Características

sociodemográficas, dados clínicos e critérios do fenótipo da fragilidade foram obtidos nas casas. A definição da fragilidade foi baseada na deteç̧ão de perda de peso, exaustão, fraqueza, lentidão e baixo nível de atividade física. Idosos com três ou mais destas características foram classificados com frágeis; com uma ou duas características como pré-frágeis. Foram calculados pontos de corte específicos para fraqueza, lentidão e baixo nível de atividade física.

\section{Resultados}

Os resultados demonstram uma diminuição de $9,5 \%$ na resistência aeróbica ao longo do tempo; de $20 \%$ na força dos membros inferiores; e a análise de resistências cruzadas observou que maior resistência aeróbica em T1 foi associada a níveis mais elevados de força corporal seis meses depois e vice-versa.

Inicialmente, os indivíduos jovens e idosos apresentavam níveis semelhantes de massa magra das pernas $(\mathrm{Y} ; 15,7 \pm 0,9$ vs $\mathrm{O} ; 13,4 \pm 1,0 \mathrm{~kg}$ ) e pico de força de extensão isométrica da perna (Y; $160 \pm 6$ vs O; $134 \pm 3$ $\mathrm{Nm}$ ). Após o repouso ao leito, a massa magra e a força da perna diminuíram em adultos idosos, mas não em adultos jovens $(\mathrm{P}<0,05)$ e foram restaurados após a reabilitação. Além disso, idosos tiveram redução na taxa de síntese de proteínas induzida por EAA e aumento de mRNA de MAFBX, p-AMPK $\alpha$ e LC3II/I $(\mathrm{P}<0,05)$. Foi concluído que os idosos são mais suscetíveis que os jovens à perda de massa muscular após um breve período de repouso no leito.

A média de idade dos participantes foi de 73,9 96,5 anos, com $64,7 \%$ de mulheres. $12,5 \%$ perderam peso no último ano, $20,5 \%$ tiveram exaustão, $17,1 \%$ lentidão, $24,5 \%$ baixo nível de atividade física e 20,5\% fraqueza. Foram considerados frágeis $9,1 \%$ e pré-frágeis $49,6 \%$. Os frágeis eram mais velhos, foram em mais consultas médicas, tiveram maior chance de internação nos últimos 12 meses e tiveram mais eventos cerebrovasculares, diabetes, neoplasias, osteoporose, incontinência fecal e urinária. Em uma população idosa independente, existem vários indivíduos frágeis e pré-frágeis. A síndrome da fragilidade foi associada com alta morbidade. Pontos de corte para fraqueza, lentidão e baixo nível de atividade física devem ser ajustados para a população em estudo. É essencial identificar idosos frágeis e pré-frágeis para intervenções apropriadas. 
Tabela 1 (continuação)

\begin{tabular}{llll}
\hline Autor e ano & Amostra & Objetivo & Método \\
$\begin{array}{lll}\text { Dutra T, Pinheiro PA, } \\
\text { Carneiro JAO et al., }\end{array}$ & $\begin{array}{l}173 \text { mulheres } \\
(\geq 60 \text { anos })\end{array}$ & $\begin{array}{l}\text { Identificar a prevalência e } \\
\text { fatores associados a sarcopenia } \\
\text { em uma população idosa } \\
\text { residente em comunidade do } \\
\text { nordeste Brasileiro. }\end{array}$ & $\begin{array}{l}\text { As associações entre } \\
\text { sarcopenia (definida através da } \\
\text { diminuição da massa e força } \\
\text { muscular e/ ou performance) }\end{array}$ \\
& & $\begin{array}{l}\text { e as variáveis independentes } \\
\text { incluindo características sócio- } \\
\text { demográficas, comportamentais } \\
\text { e condições de saúde, foram }\end{array}$ \\
& & $\begin{array}{l}\text { testadas por meio da técnica de } \\
\text { regressão logística. O nível de } \\
\text { significância adotado foi de } 5 \% .\end{array}$ \\
& &
\end{tabular}

Viana JU, Dias JMD, Batista PP et al., 2018

18 idosas sarcopênicas ( $\geq 65$ anos)

Gianoudis J, Bailey CA, Daly RM, 2015

162 idosos

(60-86 anos).

Tecchio JM, Gessinger C, 371 idosos ativos 2017
Avaliar o impacto de um programa de exercícios com carga progressiva (PECP) no desempenho muscular e funcional de idosas sarcopênicas da comunidade.

Investigar a influência do tempo assistindo à TV, tempo total sentado e a fragmentação do tempo sentado, que representa a razão entre o número de episódios sedentários dividido pelo tempo sedentário total, a massa muscular, a força, desempenho funcional $\mathrm{e}$ o risco de sarcopenia em australianos idosos residentes na comunidade.
PECP foi baseado em $75 \%$ de uma resistência máxima (12/semanas, $3 \mathrm{x} /$ semana). Medidas de desfecho: força muscular dos extensores de joelho (dinamômetro isocinético), massa muscular (dual-x ray absorptiometry DXA), desempenho funcional (Short Physical Performance Battery - SPPB). Comparações pré-pós intervenção foi por meio do teste t-test pareado.

Estudo transversal, com a intervenção de exercício físico projetado para otimizar a saúde músculoesquelética. Foram calculados antropometria, composição corporal e sarcopenia; o tempo assistindo TV (relaionado ao sedentarismo) foi auto-relatado em um questionário. Além de se utilizar o questionário de atividades físicas CHAMPS

\section{Resultados}

A prevalência de sarcopenia da população estudada foi de $17,8 \%$. A técnica de regressão logística apontou que apenas as variáveis idade avançada ( $\mathrm{p}=0,005)$ e hospitalização nos últimos 12 meses $(p=0,009)$ apresentaram significância estatística. Conclui-se que há significante prevalência de sarcopenia em idosos residente em comunidade com condições de saúde desfavoráveis; e apontou maior relação com idade superior a 80 anos e hospitalização nos últimos 12 meses

Houve aumento na potência $(p=0,01)$ e no torque $(p=0,01)$, observados nas medidas do isocinético em baixa velocidade $(60 \%)$. Observou-se também aumento no DXA $\left(5,49 \mathrm{~kg} / \mathrm{m}^{2}\right.$ vs. $\left.6,01 \mathrm{~kg} / \mathrm{m}^{2} ; \mathrm{p}=0,03\right)$ e melhora nos escores do SPPB ( 9,06 vs. 10,$28 ; p=0,01)$. O PECP aumentou o desempenho funcional e muscular das idosas sarcopênicas comunitárias.

Maior tempo de sessão global foi associado com um risco aumentado de sarcopenia; para cada incremento de $1 \mathrm{~h}$, o risco aumentou em $33 \%$ [odds ratio 1.33 (intervalo de confiança de $95 \%$ (CI) $1,05,1,68)$ ], independente da atividade física e outros fatores de estilo de vida e fatores de confusão. O tempo de visualização da TV foi associado com menor massa corporal total e massa magra das pernas após o ajuste para vários fatores de confusão e massa gorda. Houve associação entre tempo sentado total ou tempo de visualização de TV ou a taxa de fragmentação com qualquer outra medida.

Não houve associação da funcionalidade de MMSS e MMII com o IMC dos idosos ativos. Mais da metade da amostra apresentou sobrepeso. A grande maioria apresentava funcionalidade de MMSS e MMII presente. A ocupação, a auto percepção muito boa e boa de saúde e a realização de atividade física, estiveram associadas com a funcionalidade de MMII e a realização regular de exercícios físicos apresentou associação com a funcionalidade de MMSS e MMI 
Tabela 1 (continuação)

\begin{tabular}{|c|c|c|c|}
\hline Autor e ano & Amostra & Objetivo & Método \\
\hline $\begin{array}{l}\text { De Sousa KT, } \\
\text { De Mesquita LAS, } \\
\text { Pereira L A et al., } 2014\end{array}$ & $\begin{array}{l}233 \text { idosos } \\
\text { (média } 79,5 \text { anos - } \\
\mathrm{DP}=9,98)\end{array}$ & $\begin{array}{l}\text { Identificar a associação } \\
\text { entre estado nutricional e } \\
\text { dependência funcional dos } \\
\text { idosos institucionalizados de } \\
\text { Uberlândia (MG). }\end{array}$ & $\begin{array}{l}\text { Foram avaliadas variáveis } \\
\text { sociodemográficas, estado } \\
\text { nutricional e o grau de } \\
\text { dependência dos idosos. } \\
\text { Realizou-se análise multivariada } \\
\text { pela Regressão de Poisson } \\
\text { com variância robusta, para } \\
\text { verificar a associação entre o } \\
\text { estado nutricional e dependência } \\
\text { funcional. }\end{array}$ \\
\hline
\end{tabular}

Coker RH, Hays NP, Willians RH et. al., 2015

19 idosos (60-85 anos; $\mathrm{IMC}=20$ a $35 \mathrm{~kg}$ )

Cardoso RK, Araujo AM, 12 Estudos De Freitas MP et. al., 2018 experimentais com adultos com 50 anos ou mais
Examinar as mudanças na composição corporal, força muscular e estado funcional, após 10 dias de repouso no leito, e sua relação com em indivíduos mais velhos.

Revisar a literatura existente referente ao efeito do treinamento com Restrição do fluxo sanguíneo (RFS) em adultos mais velhos e idosos.
Os participantes foram submetidos a um exame físico,que incluiu linha de base, eletrocardiograma e exame de sangue. Para a avaliação da composição do corpo utilizouse uma absorciometria de raios $\mathrm{X}$ de dupla energia (DXA). Para os parametros funcionais foram realizados atividades de subida e descida em uma escada, além de um teste de caminhada por 5 minutos. Um teste ergométrico foi realizado pré e pós-repouso para determinar o $\mathrm{VO}_{2}$ pico em uma bicicleta ergométrica. Por fim, houve comparações estatísticas para todas as variáveis, incluindo antropometria, composição corporal regional, atenuação muscular, pico de $\mathrm{VO}_{2}$, utilizando testes t pareados.

Busca eletrônica nas bases de dados Pubmed/ Medline, Bireme, Scielo, Lilacs e Cochrane, publicados até dezembro de 2015. Foram incluídos estudos experimentais, publicados em inglês e português, que objetivaram verificar os efeitos do treinamento com RFS sobre qualquer desfecho em indivíduos com 50 anos ou mais. A escala Downs \& Black foi utilizada para averiguar a qualidade metodológica dos artigos.

\section{Resultados}

O baixo peso pelo Índice de Massa Corporal se associou à dependência funcional após controle para variáveis socioeconômicas e demográficas, os idosos com baixo peso tinham uma razão de prevalência de dependência de 1,20 vezes àquela dos eutróficos. Como bem reportado pela literatura, a idade também se associou à dependência, com razão de prevalência de 1,19 , para os longevos. O baixo peso constituiu importante fator associado à incapacidade funcional. Dessa forma, ressalta-se a necessidade de avaliação, monitoramento e recuperação do estado nutricional de idosos institucionalizados, que além de reduzir morbimortalidade por diversas doenças e melhorar a qualidade de vida, ainda pode prevenir e retardar a dependência funcional.

O peso e o índice de massa corporal e a massa magra das extremidades total e inferior diminuíram com o repouso no leito. Houve também reduções significativas na extensão isométrica do joelho e na extensão do joelho concêntrica de $60^{\circ}$. Além disso, houve diminuição no tempo de subida $\mathrm{e}$ descida da escada, no $\mathrm{VO}_{2}$ max, no teste de transferência de piso e no tempo de caminhada de 5 minutos.

O treinamento com RFS melhorou o grau de força, a massa, o torque e a potência muscular, a capacidade funcional, a saúde óssea, a complacência venosa, o consumo de oxigênio de pico, o fluxo sanguíneo e o equilíbrio e a performance geral. Quanto à qualidade metodológica os estudos apresentaram um escore médio de 16,2 pontos ( $\mathrm{DP}=1,6)$. A prescrição de exercícios de baixa intensidade com RFS pode ser uma alternativa para o treinamento de adultos mais velhos e idosos. 
Tabela 1 (continuação)

\begin{tabular}{|c|c|c|}
\hline Autor e ano & Amostra & Objetivo \\
\hline $\begin{array}{l}\text { Fragala MS, Fukuda DH, } \\
\text { Stout JR et al., } 2014\end{array}$ & $\begin{array}{l}25 \text { idosos } \\
(70,6 \pm 6,1 \text { anos; } \\
\mathrm{IMC}=28,1 \pm 5,4 \mathrm{~kg})\end{array}$ & $\begin{array}{l}\text { Determinar se há mudanças } \\
\text { no índice de qualidade } \\
\text { muscular (MQI) em } \\
\text { resposta ao treinamento e ao } \\
\text { destreinamento do exercício } \\
\text { resistido. }\end{array}$ \\
\hline
\end{tabular}

Shad BJ, Wallis G, Van Loon LJC et al. 2016

Da Silva SLA, Neri AL, Ferrioli E et al., 2016
Literatura internacional em língua inglesa publicada até 2016.
5532 idosos (média $73,08 \pm 6,17$ anos)
Resumir brevemente as evidências usadas para informar as recomendações atuais de saúde pública para atividade física (AF) e tempo sedentário em idosos; e discutir a presença de "resistência anabólica" em idosos, destacando a importância da $\mathrm{AF}$ regular e minimizando o comportamento sedentário

Avaliar a participação de cada item na determinação da fragilidade em idosos brasileiros

\section{Método}

Estudo longitudinal com grupo de comparação. Os voluntários foram divididos de forma aleatória em 2 grupos. $\mathrm{O}$ primeiro realizou 6 semanas de treinamento de resistência, seguido por 6 semanas de destreinamento. $\mathrm{O}$ segundo realizou 6 semanas de treinamento de resistência, além de continuar com as suas atividades diárias, seguido por 6 semanas de destreinamento. Foram medidas o índice de qualidade muscular (IQM), determinado como a potência de saída do tempo sentado (STS), massa corporal e comprimento da perna, além de avaliar a velocidade de marcha, força de preensão palmar, levantar-e-ir e massa corporal magra (MCM) avaliadas antes e após treinamento físico e destreinamento.

Foi realizada uma revisão narrativa, com base na literatura internacional em língua inglesa publicada até abril de 2016, usando as bases de dados Ovid MEDLINE (1946 a abril de 2016) e EMBASE (1974 a 10 de abril de 2016). Foram pesquisados os termos "human/humans," "old," "elder," "physical activity," "exercise," "physical activity recommendations," "physical activity guidelines," "physical inactivity," "sedentary behaviour," "sedentary time," "musculoskeletal," "health," "protein synthesis," "muscle protein synthesis," "fractional synthetic rate," "muscle protein accrual," "protein balance," "amino acid," "essential amino acid," "dietary protein," "muscle disuse," and "anabolic resistance."

A análise foi feita pela Regressão Logística Multinomial. A amostra total de 5532 idosos, selecionados aleatoriamente em diversas cidades brasileiras entre dezembro de 2008 e setembro de 2009, foi avaliada pelo fenótipo de fragilidade. Os itens mais frequentes na amostra foram o nível de atividade física, seguido da fraqueza muscular e lentidão da marcha

\section{Resultados}

$\mathrm{O}$ treinamento resistido de curto prazo melhorou de forma significativa e clinicamente a IQM (203,4 $\pm 64,31$ a $244,3 \pm 82,92 \mathrm{~W})$, o tempo de marcha $(1,85 \pm 0,36$ a $1,66 \pm 0,27 \mathrm{~s})$ e o desempenho sentado $(13,21 \pm 2,51$ a $11,05 \pm 1,58 \mathrm{~s})$. Alterações na força de preensão palmar não foram significativas ou clinicamente significativas. Destreinamento por 6 semanas não resultou em mudanças significativas em qualquer medida do desempenho pós-treino.

Os benefícios da $\mathrm{AF}$ na otimização da função musculoesquelética e da saúde metabólica estão bem estabelecidos, mas a quantidade mínima de $\mathrm{AF}$ necessária para idosos ainda é pouco definida. $\mathrm{O}$ acoplamento de $\mathrm{AF}$ e nutrição adequada é fundamental para preservar a sensibilidade anabólica muscular e, assim, manter a massa muscular esquelética e a saúde metabólica com o avançar da idade.

Os itens que apresentaram maior chance para o desenvolvimento da fragilidade foram a lentidão na marcha $(\mathrm{OR}=10,50$, IC95\% $8,55-12,90, \mathrm{p}<0,001)$ e a fraqueza muscular $(\mathrm{OR}=7,31, \mathrm{IC} 95 \%$ $6,02-8,86, p<0,001)$. O modelo com os cinco itens explicou $99,6 \%$ da fragilidade na amostra. Tais resultados sugerem que $o$ nível de atividade física, fraqueza muscular e lentidão na marcha são os itens que mais influenciam na determinação da fragilidade, mas a aplicação de todos os itens do fenótipo em conjunto é a melhor forma para a avaliação. 
Tabela 1 (conclusão)

\begin{tabular}{|c|c|c|c|c|}
\hline Autor e ano & Amostra & Objetivo & Método & Resultados \\
\hline $\begin{array}{l}\text { Confortin SC, Ono LM, } \\
\text { Barbosa AR et al., } 2018\end{array}$ & 598 idosos ( $\geq 64$ anos) & $\begin{array}{l}\text { Verificar a prevalência de } \\
\text { sarcopenia e sua associação } \\
\text { com mudanças nos } \\
\text { fatores socioeconômicos, } \\
\text { comportamentais e de saúde } \\
\text { em idosos }\end{array}$ & $\begin{array}{l}\text { Índice de massa muscular } \\
\text { apendicular (IMMA), de acordo } \\
\text { com o sexo (IMMA }<7,26 \mathrm{~kg} / \mathrm{m}^{2} \\
\text { para homens e }<5,5 \mathrm{~kg} / \mathrm{m}^{2} \text { para } \\
\text { mulheres). Foram avaliadas as } \\
\text { mudanças ocorridas entre os dois } \\
\text { momentos do estudo ( } 2009 / 2010 \\
\text { e } 2013 / 2014) \text {, relativas aos } \\
\text { fatores socioeconômicos, } \\
\text { comportamentais e de saúde. } \\
\text { Análises de regressão logística } \\
\text { bruta e ajustada. }\end{array}$ & $\begin{array}{l}\text { Prevalência de sarcopenia foi } \\
\text { de } 17 \% \text { (IC95\%: } 12,4-22,9) \text { nas } \\
\text { mulheres e de } 28,8 \% \text { (IC95\%: } \\
21,3-37,7) \text { nos homens. No } \\
\text { modelo final, as mulheres } \\
\text { que se mantiveram ingerindo } \\
\text { ou passaram a ingerir álcool } \\
\text { (OR=0,31; IC95\%: 0,11- } \\
\text { 0,91) apresentaram menor } \\
\text { chance de ter sarcopenia. } \\
\text { Aquelas que permaneceram } \\
\text { fumando ou passaram a fumar } \\
\text { (OR=2,55; IC95\%: } 1,16-5,58) \\
\text { e se mantiveram ou passaram } \\
\text { a ser insuficientemente ativas } \\
\text { (OR=2,90; IC95\%: } 1,44-5,84) \\
\text { apresentaram mais chance de } \\
\text { ter sarcopenia. Para os homens, } \\
\text { nenhuma variável de mudança } \\
\text { foi associada a sarcopenia. } \\
\text { Os resultados sugerem que } \\
\text { manter-se fumando ou passar } \\
\text { a ter o hábito de fumar, assim } \\
\text { como permanecer ou passar } \\
\text { a ser inativo fisicamente, são } \\
\text { fatores de risco preveníveis e } \\
\text { modificáveis para a sarcopenia. }\end{array}$ \\
\hline
\end{tabular}

\section{DISCUSSÃO}

A literatura descreve como os processos fisiológicos, neuroendócrinos, metabólicos e musculoesqueléticos intrínsecos ao envelhecimento podem acarretar quadros sindrômicos como a sarcopenia e o baixo peso. Ambos são características da fragilização do idoso e se correlacionam com outros fatores de ordem sociodemográfica, econômica e comportamental, acarretando vulnerabilidade.

A sarcopenia, em especial, revelou-se um dos principais fatores para risco de queda e fraturas patológicas, o que acentua vigorosamente a necessidade de intervir em sua progressão e investir em práticas preventivas.

Em um estudo transversal denominado Rede FIBRA (Perfis de Fragilidade de Idosos Brasileiros) realizado no município de Ribeirão Preto-SP, Calado et al. ${ }^{8}$ lançam mão do conceito multifatorial da chamada Síndrome de Fragilidade para perfilizar uma amostra de população idosa.

Os autores utilizam o conceito de Síndrome da Fragilidade como proposta por Fried ${ }^{9}$, onde a síndrome é caracterizada pelo declínio espiral de energia, com presença de sarcopenia, desregulação neuroendócrina e disfunção imunológica, em um ciclo vicioso que leva progressivamente à redução de energia, aumento da dependência e maior susceptibilidade a agressores. $\mathrm{O}$ fenótipo da fragilidade é diagnosticado pela sensação de fraqueza, diminuição da capacidade de realizar atividades e redução no desempenho físico, que podem resultar em incapacidade funcional. A partir de critérios tais como: (i) perda de peso não intencional: maior de $4,5 \mathrm{~kg}$ ou superior a $5 \%$ do peso corporal no último ano; (ii) diminuição da força de preensão palmar; (iii) diminuição da velocidade de marcha em segundos; (iv) exaustão e (v) baixo nível de atividade física, o diagnóstico clínico é realizado e, conforme o número de critérios presentes, os idosos passam a ser considerados como frágeis; pré-frágeis e robustos.

Conforme os dados levantados pelo estudo cerca de $12,5 \%$ dos indivíduos $(\mathrm{n}=385)$ foram positivos para perda de peso no último ano, 20,5\% apresentaram exaustão, $17,1 \%$ lentidão, 24,4\% baixa atividade física e $20,5 \%$ fraqueza. Sendo a baixa atividade física o critério mais prevalente para a síndrome de fragilidade, principalmente no que tange à perda da massa muscular e desencadeamento do ciclo de Fried'.

Uma extensão do mesmo estudo conduzido por Da Silva et al. ${ }^{10}$ em diversos municípios brasileiros (Barueri-SP, Belém-PA, Belo Horizonte-MG, Campinas-SP, Cuiabá-MT, Ermelindo Matarazzo-SP, 
Fortaleza-CE, Ivotí-RS, Juiz de Fora-MG, Parnaíba-PI, Poços de Caldas-MG, Recife-PE, Ribeirão Preto-SP e Santa Cruz-RN) corrobora o dado de que o baixo nível de atividade física é o componente mais comum na amostra total atingindo cerca de $27,5 \%(n=5532)$.

Baixo nível de atividade física $\left(\mathrm{R}^{2}=0,37\right)$, lentidão da marcha $\left(\mathrm{R}^{2}=0,32\right)$ e força muscular $\left(\mathrm{R}^{2}=0,28\right)$ foram os itens que, isoladamente, tiveram maior poder de explicação para a fragilidade, dentro de modelos individuais ${ }^{10}$. Já a perda de peso, isoladamente, teve valores menos significativos para o desenvolvimento de fragilidade.

Quanto à capacidade funcional do idoso e a autonomia para executar tarefas De Sousa et al. ${ }^{11}$, em um estudo transversal, descreveram o baixo peso como o componente de maior proporção de risco para dependência funcional.

O baixo peso representou, isoladamente, um importante fator de risco para a dependência dos idosos, uma vez que é necessária massa corporal preservada para a realização de qualquer tarefa que exija esforço físico como, por exemplo, as Atividades de vida diária (AVD). Os autores, no entanto, declaram que a ausência de dados a respeito de sarcopenia foi uma das limitações do estudo e ressalta a importância de implementar ações preventivas ou retardatárias do declínio funcional.

Existem, contudo, controversas na literatura a respeito da associação entre capacidade funcional e IMC. Tecchio e Gessinger ${ }^{12}$ demonstram, em um estudo transversal, que o IMC não apresenta correlação com a funcionalidade de membros superiores e inferiores em idosos. Isto contradiz a informação do estudo anteriormente citado.

Esta divergência talvez possa ser explicada pela heterogeneidade das amostras. O estudo elaborado por De Sousa et al. ${ }^{11}$, contava com uma população de idosos institucionalizados e, portanto, mais propensos a fragilização. Já segundo Tecchio e Gessinger ${ }^{12}$, possuía uma amostra de idosos fisicamente ativos.

Embora divirjam no quesito IMC, Tecchio e Gessinger ${ }^{12}$ encontraram relação positiva entre prática de exercício físico e manutenção da capacidade funcional além de uma correlação entre a perda progressiva de massa muscular no idoso, decorrente do envelhecimento, e o risco duas a três vezes maior de comprometimento funcional. Os autores concluíram que a prática de exercícios é elemento fundamental para preservar a função locomotora, a massa muscular e óssea nos indivíduos mais velhos.

Coker et al. $^{13}$, a partir de uma intervenção de curto prazo, mostraram que após dez dias de repouso no leito, os idosos apresentaram redução significativa de peso corporal, do índice de massa corporal e da massa magra total e dos membros inferiores, levando a um aumento de gordura corporal total e diminuição da força muscular, bem como do pico de $\mathrm{VO}_{2}$. Como consequência, observou-se lentidão da velocidade de marcha, incapacidade de realizar tarefas diárias e maior risco de mortalidade. Diante disto, o autor reforça a importância de programas de mobilidade para reverter tais consequências prejudiciais.

Já os autores Tanner et al. ${ }^{14}$, em estudo semelhante, demonstraram que a perda de tecido magro e força muscular é dependente da idade e concluíram que idosos são mais suscetíveis à perda de massa muscular que jovens. Após cinco dias de repouso no leito, observou-se somente em idosos a diminuição de 3\% do peso corporal e de $4 \%$ da massa magra total, além de $4 \%$ da massa muscular dos membros inferiores e 16\% do alongamento muscular (força isométrica). Em nível celular, observaram atenuação das taxas de síntese protéica e aumento dos marcadores de proteólise muscular (AMPK $\alpha$, MAFBX e LC3II/I). Com isso, depreenderam que a população com idade mais avançada tem maior probabilidade de deteriorar a massa muscular durante repouso em curto prazo, devido à desregulação do anabolismo proteico. $\mathrm{O}$ estudo prosseguiu com um programa estruturado de reabilitação com treinamento de resistência combinado à suplementação proteica. Como resultado houve reversão dos déficits funcionais musculares e restaurouse a sensibilidade anabólica aos níveis pré-leitos.

Em estudo transversal, Gianoudis, Bailey e Daly ${ }^{15}$ determinaram que para cada incremento de $1 \mathrm{~h}$ no tempo de permanência diária na posição sentado houve um aumento de 33\% no risco de apresentar sarcopenia. Um exame adicional de associação entre comportamentos sedentários revelou que maior tempo de TV diário, possivelmente combinado ao hábito de petiscar, está relacionado com menores índices de massa corporal total e massa magra de membro inferior. Este resultado aponta que o comportamento sedentário está associado ao aumento das chances de desenvolver sarcopenia.

Ainda sobre o impacto do estilo de vida sedentário, Magistro et al. ${ }^{16}$ examinaram sua influência sobre a resistência aeróbica e força dos membros inferiores ao longo do tempo em uma amostra de idosos italianos sedentários e notaram diminuição relativa de 9,5\% da resistência aeróbica e de $20 \%$ da força corporal inferior no período de um ano. Tais fatores demonstraram correlação de modo que maior resistência no início do estudo predizia maior força corporal ao final de um ano, enquanto maior força influenciou a resistência aeróbica apenas nos primeiros seis meses. Os autores enfatizam, 
em sua discussão, a importância de se projetar um treinamento físico específico para idosos e afirmam que há um declínio da resistência aeróbica e da força ao longo do tempo, sendo interessante a atividade física como fator de proteção para boas condições físicas e psicológicas, sobretudo em indivíduos frágeis.

Outro estudo longitudinal tomou como referência a população de uma pesquisa epidemiológica intitulada Condições de Saúde de Idosos de Florianópolis - EpiFloripa Idoso $^{17}$ e avaliou a prevalência da sarcopenia diante das seguintes variáveis: socioeconômicas (condições de trabalho), comportamentais (alimentação, uso de álcool, tabagismo e atividade física) e saúde (morbidades, cognição, histórico de quedas, saúde mental e mobilidade).

Neste trabalho, Confortin et al. ${ }^{17}$ constataram que idosas mantendo um estilo de vida sedentário aumentam o risco de desenvolver sarcopenia $(\mathrm{OR}=2,10$; IC95\%: 1,07-4,12). Em relação a atividade física, mulheres insuficientemente ativas apresentaram chances mais elevadas de desenvolver sarcopenia. Isto ocorre como resultado das alterações celulares induzidas pelo baixo recrutamento muscular, o que leva a atrofia e deterioração da capacidade contrátil dos miócitos. Estes processos são ainda mais acentuados pela senescência natural das fibras musculares. Vale ressaltar, que mesmo mulheres ativas que passaram a ter estilo de vida inativo apresentaram risco para desenvolver sarcopenia, o que reforça a importância de manter um estilo de vida ativo durante toda a vida e, sobretudo, na velhice.

Estes achados foram reiterados por Dutra et al. ${ }^{18}$, cujo estudo de prevalência e fatores associados à sarcopenia em idosas residentes da zona urbana do município de Lafaiete Coutinho - BA constatou que as idosas com idade mais avançada, sobretudo $\geq 80$ anos, e insuficientemente ativas possuem maior probabilidade de apresentar sarcopenia.

O estudo experimental conduzido por Viana, Dias, Batista et al. ${ }^{19}$ observou melhorias significativas na massa magra corporal, força muscular e desempenho físico de idosas sarcopênicas após 12 semanas em um Programa de Exercícios Resistidos (PREP). Houve um incremento de $0,52 \mathrm{~kg} / \mathrm{h}^{2}$ de massa magra após o PREP constatado por exame de imagem.

Os autores citam diversos outros trabalhos que corroboram seus achados, sobretudo no aspecto de ganho de massa muscular e explica que essas mudanças ocorreram devido ao fato de ter havido maior estimulação muscular, aumentando a demanda por recrutamento de fibras. Esse estímulo pode ter sido efetivo para o recrutamento de fibras, principalmente do tipo I, devido à diferença na força muscular. (pico de torque) demonstrado a baixa velocidade $\left(60^{\circ} / \mathrm{s}\right)$. A literatura indica que na fisiologia da contração muscular, durante a avaliação por isocinética, velocidades mais lentas tendem a demonstrar mais força, provavelmente devido ao maior recrutamento de fibras do tipo $\mathrm{I}^{19}$.

Mencionam ainda, Viana et al. ${ }^{19}$, que, embora haja predominância de fibras do tipo I e perda expressiva de fibras tipo II na sarcopenia, há necessidade de estudos futuros para identificar o tipo de fibra recrutada mediante o programa de exercícios resistidos. Isto se deve ao fato de que houve ganho de massa muscular, força e função, sendo as duas ultimas características das fibras do tipo II.

Fragala et al. ${ }^{20}$ propuseram um programa semelhante para uma amostra de idosos. A intervenção consistia em 6 semanas de treinamento resistido. Ao final do programa, verificou-se melhora na capacidade funcional, velocidade de marcha e movimento sentar/ levantar. Contudo, não foi observado incremento na massa corporal magra ou na força. Deve-se levar em consideração, no entanto, que o tempo de intervenção deste estudo em relação ao de Viana et al. é mais reduzido, o que pode ter impactado nos resultados.

Em uma revisão sistemática, Cardoso, Araújo, De Freitas et. al. ${ }^{21}$ discutem os efeitos do Treinamento com Restrição Parcial do Fluxo Sanguíneo (RPF) em idosos. Este tipo de exercício, segundo descrevem, utiliza uma banda inflável que bloqueia parcialmente o fluxo sanguíneo na raiz dos membros. Isto otimizaria os resultados uma vez que proporcionaria exercícios com volumes e intensidades inferiores, portanto, menos estresse mecânico. Estas características o tornam um método ideal para populações fragilizadas e impossibilitadas de praticar atividades de alta intensidade e impacto, como idosos com baixo peso e sarcopenia.

Dentre os desfechos mais estudados das respostas crônicas do treinamento com RPF em adultos mais velhos, a força muscular foi considerada em sete artigos e hipertrofia muscular em cinco artigos. Os resultados encontrados pelos estudos revisados indicaram que o treinamento de baixa intensidade foi eficaz para aumentar a força e promover a hipertrofia muscular quando realizada com RPF 21 .

Estes resultados, explicam os autores, se devem às adaptações neuromusculares que ocorrem tais como melhora na coordenação neuromuscular e/ou nervosa, recrutamento de fibras rápidas e de suas unidades motoras, além de maior ativação muscular. Além das respostas neuromusculares, o processo de hipertrofia e aumento da força muscular promovido pelo treinamento com RPF é devido às respostas metabólicas e endócrinas. $\mathrm{O}$ estresse metabólico promovido 
pela hipóxia associada ao exercício leva à translocação do transportador de glicose tipo 4 (GLUT-4), estimulando a absorção de glicose muscular. Além disso, o exercício com RPF proporciona uma duração mais longa da acidose metabólica, que estimula a liberação sistêmica do hormônio do crescimento $(\mathrm{GH})$ e o consequente aumento da liberação do fator de crescimento insulina-1 (IGF-1) ${ }^{21}$.

Outro benefício desta modalidade de exercício descrito no trabalho é que não houve mudanças inflamatórias adicionais mediante o treinamento com $\mathrm{RPF}$, algo imprescindível considerando a fisiologia do idoso com baixo peso e sarcopênico.

Os autores, Shad, Wallis, Van Loon et al. ${ }^{22}$, em revisão narrativa, identificaram que a maioria das evidências atuais apontam que a participação regular de idosos em atividades físicas de moderada a vigorosa intensidade é fundamental para preservar a massa muscular esquelética, a força e a função física.

Segundo esses autores, estas práticas podem isoladamente prevenir o surgimento de condições debilitantes como a sarcopenia e se combinadas a nutrição adequada podem potencializar a sensibilidade anabólica muscular e otimizar a saúde metabólica.

\section{CONCLUSÃO}

O exercício físico foi associado à manutenção da capacidade funcional, preservação da massa magra corporal, diminuição do risco de desenvolver sarcopenia e fragilidade na população idosa. Exercícios resistidos ou com bloqueio parcial de fluxo sanguíneo apresentaram eficácia no ganho de força e massa muscular.

Inversamente, o estilo de vida sedentário revelou-se um fator de risco para o desenvolvimento de síndrome de fragilidade, sarcopenia e declínio funcional do idoso.

Embora a literatura divirja em relação ao impacto do baixo peso na capacidade funcional do idoso, este fator é descrito como componente da síndrome de fragilidade, assim como o baixo nível de atividade física. Dito isto, os estudos demonstram que este segundo fator é um dos mais prevalentes dentre a população de idosos frágeis.

Os benefícios de manter níveis satisfatórios de atividade física na população idosa abrangeram, principalmente, os aspectos neuromusculares e metabólicos que acarretam a sarcopenia e a depleção e massa muscular no idoso. Sendo o exercício físico um fator protetor contra estes processos.

Houve, contudo, poucos dados que relacionassem isoladamente o impacto da atividade física sobre a variável baixo peso. A única relação estabelecida foi a de que a prática de exercícios promove a manutenção da massa corporal magra e potencializa o anabolismo proteico.

Evidentemente, não somente a atividade física, mas os aspectos sociodemográficos, econômicos, comportamentais e genéticos devem ser levados em consideração na construção de intervenções voltadas para a prevenção de sarcopenia e de fatores predisponentes, tais como o baixo peso, na população idosa.

\section{REFERÊNCIAS}

1. Assumpção D, Borim FSA, Francisco PMSB, Néri AL. Fatores associados a baixo peso em idosos comunitários em sete cidades brasileiras: estudos FIBRA. Ciênc Saúde Coletiva. 2018;23(4):1143-50. https://doi. org/10.1590/1413-81232018234.17422016

2. Cançado F, Doll J, Gorzoni M. Tratado de geriatria e gerontologia. 4. ed. Rio de Janeiro: Guanabara Koogan; 2016.

3. Cruz-Jentoft AJ, Bahat G, Bauer J, Boirie Y, Bruyère $O$, Cederholm T, Cooper C, Landi F, Rolland Y, Sayer AA, Schneider SM, Sieber CC, Topinkova E, Vandewoude M, Visser M, Zamboni M; Writing Group for the European Working Group on Sarcopenia in Older People 2 (EWGSOP2), and the Extended Group for EWGSOP2. Sarcopenia: revised European consensus on definition and diagnosis. Age Ageing. 2019;48(1):16-31. https://doi. org/10.1093/ageing/afz046

4. Martinez B, Camelier FWR, Camelier A. Sarcopenia em idosos: um estudo de revisão. RPF. 2014;4(1):62-70. https://doi.org/10.17267/2238-2704rpf.v4i1.349

5. Miranda VPN, Peluzio MCG, Franceschini SCC, Priore SE. Marcadores inflamatórios na avaliação nutricional: relação com parâmetros antropométricos, composição corporal e níveis de atividade física. RASBRAN. 2014;6(1):61-72.

6. Lebrão ML, Duarte Y, organizadores. O projeto SABE no município de São Paulo: uma abordagem inicial. Brasília: OPAS/MS; 2003.

7. Abreu DROM, Novais ES, Oliveira RR, Mathias TAF, Marcon SS. Internação e mortalidade por quedas em idosos no Brasil: análise de tendência. Ciênc Saúde Coletiva. 2018;23(4):1131-41. https://doi. org/10.1590/1413-81232018234.09962016

8. Calado LB, Ferriolli E, Moriguti JC, Martinez EZ, Lima NKC. Síndrome da fragilidade em uma população urbana independente no Brasil (estudo FIBRA): um estudo transversal populacional. Sao Paulo Med. J. 2016;134(5):385-92. https://doi.org/10.1590/15163180.2016.0078180516

9. Fried LP, Tangen CM, Walston J, Newman AB, Hirsch C, Gottdiener J, Seeman T, Tracy R, Kop WJ, Burke G, McBurnie MA; Cardiovascular Health Study Collaborative Research Group. Frailty in older adults: evidence for a phenotype. J Gerontol A Biol Sci Med Sci. 2001;56(3):M146-56. https://doi.org/10.1093/ gerona/56.3.m146 
10. Silva SLA; Neri AL, Ferrioli E, Lourenço RA, Dias RC. Fenótipo de fragilidade: influência de cada item na determinação da fragilidade em idosos comunitários Rede Fibra. Ciênc Saúde Coletiva. 2016;21(11):3483-92. https://doi.org/10.1590/1413-812320152111.23292015

11. Sousa KT, Mesquita LAS, Pereira LA, Azevedo CM. Baixo peso e dependência funcional em idosos institucionalizados de Uberlândia (MG), Brasil. Ciênc Saúde Coletiva. 2014;19(8):3513-20. https://doi. org/10.1590/1413-81232014198.21472013

12. Tecchio JM, Gessinger C. Upper and lower limb functionality and body mass index in physically active older adults. Fisioter Mov. 2017;30(suppl. 1):45-54. https://doi.org/10.1590/1980-5918.030.s01.ao04

13. Coker RH, Hays NP, Willians RH, Wolfe RR, Evans WJ. Bed rest promotes reductions in walking speed, functional parameters, and aerobic fitness in older, healthy adults. J Gerontol A Biol Sci Med Sci. 2015;70(1):91-6. https://doi.org/10.1093/gerona/glu123

14. Tanner RE, Brunker LB, Agergaard J, Barrows KM, Briggs RA, Kwon OS, Young LM, Hopkins PN, Volpi E, Marcus RL, Lastayo PC, Drummond MJ. Age-related differences in lean mass, protein synthesis and skeletal muscle markers of proteolysis after bed rest and exercise rehabilitation. J Physiol. 2015;593(18):4259-73. https://doi.org/10.1113/jp270699

15. Gianoudis J, Bailey CA, Daly RM. Associations between sedentary behaviour and body composition, muscle function and sarcopenia in community-dwelling older adults. Osteoporos Int. 2015;26(2):571-9. https://doi. org/10.1007/s00198-014-2895-y

16. Magistro D, Candela F, Brustio PR, Liubicich ME, Rabaglietti E. A longitudinal study on the relationship between aerobic endurance and lower body strength in Italian sedentary older adults. J Aging Phys Act. 2015; 23(3):444-51. https://doi.org/10.1123/japa.20130215

17. Confortin SC, Ono LM, Barbosa AR, D'orsi E. Sarcopenia e sua associação com mudanças nos fatores socioeconômicos, comportamentais e de saúde: Estudo EpiFloripa Idoso. Cad Saúde Pública. 2018;34(12):e00164917. https://doi.org/10.1590/0102. $311 \times 00164917$

18. Dutra T, Pinheiro PA, Carneiro JAO, Coqueiro RS, Fernandes MH. Prevalence and factors associated with sarcopenia in elderly women living in the community. Rev Bras Cineantropom Desempenho Hum. 2015;17(4): 460-71. https://doi.org/10.5007/1980-0037.2015v17n4p460

19. Viana JU, Dias JMD, Batista PP, Silva SLA, Dias RC, Lustosa LP. Effect of a resistance exercise program for sarcopenic elderly women: quasi-experimental study. Fisioter Mov. 2018;31:e003111. https://doi. org/10.1590/1980-5918.031.ao11

20. Fragala MS, Fukuda DH, Stout JR, Townsend JR, Emerson NS, Boone CH, Beyer KS, Oliveira LP, Hoffman JR. Muscle quality index improvex with resistance exercise training in older adults. Exp Gerontol. 2014;53:1-6. https://doi.org/10.1016/j.exger.2014.01.027
21. Cardoso RK, Araujo AM, Freitas MP, Rombaldi AJ. Effect of training with partial blood flow restriction in older adults: a systematic review. Rev Bras Cineantropom Hum. 2018;20(2):2019-28.

22. Shad BJ, Wallis G, van Loon LJC, Thompson JL. Exercise prescription for the older population: the interactions between physical activity, sedentary time, and adequate nutrition in maintaining musculoskeletal health. Maturitas. 2016;93:78-82. https://doi.org/10.1016/j. maturitas.2016.05.016

\section{AUTORES}

MARIA Elisa GONZALEZ MANSO

Doutora em Ciências Sociais, pós-doutorado e mestrado em Gerontologia Social PUC-SP. Master em Psicogerontologia pela Universidade Maimônides, Buenos Aires, Argentina. Médica. Professora titular dos cursos de medicina do Centro Universitário São Camilo. Orientadora docente da Liga de Estudos do Processo de Envelhecimento LEPE, São Paulo, SP, Brasil.

E-mail: mansomeg@hotmail.com

Orcid: https://orcid.org/0000-0001-5446-233X

DEBORAH LORENZO GOMEZ SILVA

Musicoterapeuta. Graduanda do curso de medicina do Centro Universitário São Camilo (CUSC) e membro da Liga de Estudos do Processo de Envelhecimento (LEPE) São Paulo, SP, Brasil.

E-mail: deborah.lorenzo89@gmail.com

Orcid: https://orcid.org/0000-0003-1419-7599

RAFAELA RAMOS D'OlIVEIRA

Graduanda do curso de medicina do Centro Universitário São Camilo (CUSC) e membro da Liga de Estudos do Processo de Envelhecimento (LEPE), São Paulo, SP, Brasil.

E-mail: rafaelaramos11@hotmail.com

Orcid: https://orcid.org/0000-0002-7952-5277

BEATRIZ MYUNG JIN KIM

Graduanda do curso de medicina do Centro Universitário São Camilo (CUSC) e membro da Liga de Estudos do Processo de Envelhecimento (LEPE), São Paulo, SP, Brasil.

E-mail: biaaak.97@gmail.com

Orcid: https://orcid.org/0000-0002-2085-1414

ANA FLÁVIA DE LIMA SANTOS

Graduanda do curso de nutrição do Centro Universitário São Camilo (CUSC)

e membro da Liga de Estudos do Processo de Envelhecimento (LEPE), São Paulo, SP, Brasil.

E-mail: anaflavia0695@gmail.com

Orcid: https://orcid.org/0000-0003-1646-1402

EDUARDO ANTONIO BARROS SILVA JUNIOR

Graduando do curso de medicina do Centro Universitário São Camilo (CUSC) e membro da Liga de Estudos do Processo de Envelhecimento (LEPE), São Paulo, SP, Brasil.

E-mail: eduardoantoniojunior@hotmail.com

Orcid: https://orcid.org/0000-0002-4453-7366 